\title{
CONDUCTION OF HEAT IN REGIONS BOUNDED BY PLANES AND CYLINDERS
}

\author{
J. C. JAEGER
}

1. Introduction. The Green's functions for regions bounded by surfaces of the cylindrical coordinate system are well known. ${ }^{1}$ From them solutions may be obtained for problems in which the initial temperature is zero and the surfaces are kept at temperatures which are known functions of the coordinates; the application of Green's function in regions extending to infinity has not been completely studied and the conditions to be satisfied by functions prescribed in such regions are not known. An alternative method of solving such problems consists of using the Laplace transformation and solving the resulting subsidiary equation by separation of variables; here it is found necessary to assume that temperatures given on surfaces extending to infinity satisfy very narrow conditions, such as those of Fourier's or Weber's integral theorems. This is illustrated in $\$ 3$. Problems of conduction of heat in regions bounded by cylinders and planes, some of which extend to infinity, and with constant surface (and initial) temperatures, or with a radiation boundary condition

$$
\frac{\partial v}{\partial n}+h\left(v-v_{0}\right)=0
$$

at a surface, are of considerable importance and the constant surface temperatures do not satisfy the conditions referred to above [cf. §3]. The method given below gives a simple solution of all such problems; the results given form a complete set from which the solutions of all temperature problems in solids bounded by a cylinder and planes perpendicular to its axis, with constant surface (and initial) temperatures, can be written down. Problems involving a radiation boundary condition at some of the surfaces, and problems on the hollow cylinder, may be solved in the same way.

The method was suggested by that given in a previous note ${ }^{2}$ which consisted of the use of a double Laplace transformation; this gives a solution under very wide conditions on the surface temperatures, roughly that they be of exponential type in the space variable. This method also allows verification that the solution obtained does satisfy

\footnotetext{
${ }^{1}$ Carslaw, Conduction of Heat (2d edition, 1921) chaps. 9 and 10; for a discussion using the Laplace transformation see Carslaw and Jaeger, Journal of the London Mathematical Society, vol. 15 (1940), p. 273.

${ }^{2}$ This Bulletin, vol. 46 (1940), pp. 687-693. This paper will be referred to as I.
} 
the differential equation and boundary conditions. In I it was applied to problems on the semi-infinite strip but it may also be applied to problems involving cylindrical boundaries. The form of the solutions obtained by this process suggests a simple method of solving problems of the types referred to above. This is here applied to obtain solutions for regions bounded by surfaces of the cylindrical coordinate system with constant surface temperatures. In $\$ 2$ one such problem is solved in detail to illustrate the method, and in $\$ 3$ the solution is compared with that obtained by direct separation of variables. Other problems are solved briefly in $\S \S 4-7$.

The method given here is formal both in the application of the Laplace transformation and in that infinite processes are interchanged without justification; in special cases it can be verified directly that the results do satisfy the differential equations and initial and boundary conditions; as remarked above this is best done by the method of I, when applicable. The use of the Laplace transformation and the method of evaluating and justifying solutions by the use of the inversion theorem followed by contour integration will be assumed known ${ }^{3}$ and used freely without comment.

2. Heat conduction in the semi-infinite cylinder $z>0,0 \leqq r<a$; $r=a$ maintained at unit temperature for $z>0$ and $t>0 ; z=0$ maintained at zero for $0 \leqq r<a, t>0$; the initial temperature zero. We have to solve

$$
\frac{\partial^{2} v}{\partial r^{2}}+\frac{1}{r} \frac{\partial v}{\partial r}+\frac{\partial^{2} v}{\partial z^{2}}-\frac{1}{\kappa} \frac{\partial v}{\partial t}=0, \quad 0 \leqq r<a, z>0, t>0,
$$

with

$$
\begin{array}{lll}
v=1, & r=a, & z>0, t>0 ; \\
v=0, & z=0, & 0 \leqq r<a, t>0 ; \\
v=0, & t=0, & z>0,0 \leqq r<a .
\end{array}
$$

We multiply (1) and its boundary conditions (2) and (3) by $e^{-p t}$, $R(p)>0$, then writing

$$
\bar{v}(\dot{p})=\int_{0}^{\infty} e^{-p t} t_{v}(t) d t
$$

for the Laplace transform of $v$ we obtain the subsidiary equation

\footnotetext{
${ }^{3}$ Cf. Carslaw and Jaeger, Proceedings of the Cambridge Philosophical Society, vol. 35 (1939), p. 394, and this Bulletin, vol. 45 (1939), p. 407.
} 


$$
\frac{\partial^{2} \bar{v}}{\partial r^{2}}+\frac{1}{r} \frac{\partial \bar{v}}{\partial r}+\frac{\partial^{2} \bar{v}}{\partial z^{2}}-q^{2} \bar{v}=0, \quad 0 \leqq r<a, z>0,
$$

where

$$
q^{2}=p / \kappa
$$

This notation will be used throughout. Equation (6) is to be solved with boundary conditions

$$
\begin{aligned}
& \bar{v}=1 / p, \quad r=a, \quad z>0 ; \\
& \bar{v}=0, \quad z=0, \quad 0 \leqq r<a .
\end{aligned}
$$

Equation (6) is satisfied by terms of type

$$
J_{0}\left(r \alpha_{m}\right) e^{-z\left(q^{2}+\alpha_{m}^{2}\right)^{1 / 2}}
$$

where $\alpha_{m}, m=1,2, \cdots$, are the positive roots of

$$
J_{0}(a \alpha)=0 .
$$

As suggested by the form of the results obtained in I, we seek a solution of (6) of type

$$
\frac{1}{p} \frac{I_{0}(q r)}{I_{0}(q a)}+\sum_{m=1}^{\infty} A_{m} J_{0}\left(r \alpha_{m}\right) e^{-z\left(q^{2}+\alpha_{m}^{2}\right)^{1 / 2}}
$$

in which the first term is the solution of the subsidiary equation for the infinite cylinder with unit surface temperature and the second a series of correcting terms which satisfy (6) and vanish when $r=a$, and whose coefficients $A_{m}$ are to be chosen so that (12) satisfies (9). This requires

$$
\frac{1}{p} \frac{I_{0}(q r)}{I_{0}(q a)}+\sum_{m=1}^{\infty} A_{m} J_{0}\left(r \alpha_{m}\right)=0, \quad 0 \leqq r<a,
$$

that is,

Thus

$$
A_{m}=-\frac{2 \alpha_{m}}{a p\left(q^{2}+\alpha_{m}^{2}\right) J_{1}\left(a \alpha_{m}\right)}
$$

$$
\bar{v}=\frac{I_{0}(q r)}{p I_{0}(q a)}-\frac{2}{a} \sum_{m=1}^{\infty} \frac{\alpha_{m}}{p\left(q^{2}+\alpha_{m}^{2}\right)} \frac{J_{0}\left(r \alpha_{m}\right)}{J_{1}\left(a \alpha_{m}\right)} e^{-z\left(\alpha_{m}^{2}+q^{2}\right)^{1 / 2}}
$$

satisfies the subsidiary equation (6) and its boundary conditions (8) and (9). As remarked in $\$ 1$ this result is obtained directly by the method of I. 
From (14) $v(t)$ is found in the usual way ${ }^{4}$ by the use of the inversion theorem for the Laplace transformation and we have finally

$$
\begin{aligned}
v(t)= & \frac{1}{2 \pi i} \int_{\gamma-i \infty}^{\gamma+i \infty} e^{\lambda t} \bar{v}(\lambda) d \lambda=1-\frac{2}{a} \sum_{m=1}^{\infty} \frac{J_{0}\left(r \alpha_{m}\right) e^{-z \alpha_{m}}}{\alpha_{m} J_{1}\left(a \alpha_{m}\right)} \\
& -\frac{4}{\pi a} \sum_{m=1}^{\infty} \frac{\alpha_{m} J_{0}\left(r \alpha_{m}\right)}{J_{1}\left(a \alpha_{m}\right)} \int_{0}^{\infty} \frac{e^{-\kappa t\left(u^{2}+\alpha_{m}^{2}\right)} \sin z u d u}{u\left(u^{2}+\alpha_{m}^{2}\right)} .
\end{aligned}
$$

This may easily be put in the more suitable form for computation

$$
\begin{aligned}
v=1-\frac{1}{a} \sum_{m=1}^{\infty} \frac{J_{0}\left(r \alpha_{m}\right)}{\alpha_{m} J_{1}\left(a \alpha_{m}\right)}\{ & 2 e^{-\kappa \alpha_{m}^{2} t} \operatorname{erf} \frac{z}{2(\kappa t)^{1 / 2}} \\
& +e^{z \alpha_{m}} \operatorname{erfc}\left[\frac{z}{2(\kappa t)^{1 / 2}}+\alpha_{m}(\kappa t)^{1 / 2}\right] \\
& \left.+e^{-z \alpha_{m}} \operatorname{erfc}\left[\frac{z}{2(\kappa t)^{1 / 2}}-\alpha_{m}(\kappa t)^{1 / 2}\right]\right\} .
\end{aligned}
$$

3. Comparison of the solution of $\$ 2$ with that obtained formally by direct separation of variables in the subsidiary equation. Consider the problem of $\$ 2$ but with $r=a$ maintained at $f(z)$ in place of unity for $t>0, z>0$. The subsidiary equation (6) has to be solved with boundary condition (9) and with

$$
\bar{v}=\frac{1}{p} f(z), \quad r=a, z>0 .
$$

We seek a solution composed of terms of type $I_{0}\left(q^{\prime} r\right) \sin u z$, where

$$
q^{\prime}=\left(q^{2}+u^{2}\right)^{1 / 2} \text {. }
$$

Such a solution will be

$$
\bar{v}=\int_{0}^{\infty} g(u) I_{0}\left(q^{\prime} r\right) \sin u z d u
$$

This satisfies (6) and (9), and (17) requires

$$
\int_{0}^{\infty} g(u) I_{0}\left(q^{\prime} a\right) \sin u z d u=\frac{1}{p} f(z) .
$$

If we assume that $f(z)$ satisfies conditions for Fourier's integral theorem, for example, $f(z)$ satisfies Dirichlet's conditions and $\int_{0}^{\infty} f(z) d z$ converges absolutely, $g(u)$ can be determined and we obtain

${ }^{4}$ Cf. Carslaw and Jaeger, loc. cit. 


$$
\bar{v}=\frac{2}{\pi} \int_{0}^{\infty} \frac{I_{0}\left(r q^{\prime}\right)}{p I_{0}\left(a q^{\prime}\right)} \sin u z d u \int_{0}^{\infty} f\left(z^{\prime}\right) \sin u z^{\prime} d z^{\prime},
$$

and hence, using the inversion theorem for the Laplace transformation,

$$
\begin{array}{r}
v=\frac{2}{\pi} \int_{0}^{\infty}\left\{\frac{I_{0}(r u)}{I_{0}(a u)}-\frac{2}{a} \sum_{m=1}^{\infty} \frac{\alpha_{m} e^{-\kappa\left(\alpha_{m}^{2}+u^{2}\right) t} J_{0}\left(r \alpha_{m}\right)}{\left(\alpha_{m}^{2}+u^{2}\right) J_{1}\left(a \alpha_{m}\right)}\right\} \sin u z d u \\
\cdot \int_{0}^{\infty} f\left(z^{\prime}\right) \sin u z^{\prime} d z^{\prime},
\end{array}
$$

where the $\alpha_{m}, m=1,2, \cdots$, are the positive roots of $J_{0}(a \alpha)=0$ and the method of solution is available only for the above restricted values of $f(z)$. However, if we invert the orders of integration in (19) convergent solutions may be obtained for functions $f(z)$ which do not satisfy the conditions above, for example (15) may be obtained in this way.

4. The region $r>a, 0<z<l ; z=l$ kept at unity for $t>0$; the other surfaces at zero; the initial temperature zero. Here the subsidiary equation is

$$
\frac{\partial^{2} \bar{v}}{\partial r^{2}}+\frac{1}{r} \frac{\partial \bar{v}}{\partial r}+\frac{\partial^{2} \bar{v}}{\partial z^{2}}-q^{2} \bar{v}=0, \quad r>a, 0<z<l
$$

to be solved with

$$
\begin{aligned}
& \bar{v}=1 / p, \quad z=l, \quad r>a ; \\
& \bar{v}=0, \quad z=0, \quad r>a ; \\
& \bar{v}=0, \quad r=a, \quad 0<z<l .
\end{aligned}
$$

The solution of the subsidiary equation for the infinite flat plate $0<z<l$ with boundary conditions $(22)$ and $(23)$ is $(\sinh q z) /(p \sinh q l)$, so we choose for the solution of (21)

$$
\bar{v}=\frac{\sinh q z}{p \sinh q l}+\sum_{n=1}^{\infty} A_{n} \sin \frac{n \pi z}{l} K_{0}\left(r q_{n}\right)
$$

where

$$
q_{n}=\left(q^{2}+n^{2} \pi^{2} / l^{2}\right)^{1 / 2} .
$$

This satisfies (21), (22), and (23), and (24) requires

$$
\frac{\sinh q z}{p \sinh q l}+\sum_{n=1}^{\infty} A_{n} \sin \frac{n \pi z}{l} K_{0}\left(a q_{n}\right)=0
$$


that is,

Thus

$$
A_{n}=\frac{2 n \pi(-)^{n}}{p l^{2} q_{n}^{2} K_{0}\left(a q_{n}\right)}
$$

$$
\bar{v}=\frac{\sinh q z}{p \sinh q l}+\frac{2 \pi}{p l^{2}} \sum_{n=1}^{\infty} \frac{n(-)^{n} K_{0}\left(r q_{n}\right)}{q_{n}^{2} K_{0}\left(a q_{n}\right)} \sin \frac{n \pi z}{l} .
$$

And, using the inversion theorem for the Laplace transformation,

$$
\begin{aligned}
v= & \frac{z}{l}+\frac{2}{\pi} \sum_{n=1}^{\infty} \frac{(-)^{n} K_{0}(n \pi r / l)}{n K_{0}(n \pi a / l)} \sin \frac{n \pi z}{l} \\
& -\frac{4}{l^{2}} \sum_{n=1}^{\infty}\left\{n(-)^{n} e^{-\kappa n^{2} \pi^{2} t / l^{2}} \sin \frac{n \pi z}{l}\right. \\
& \left.\int_{0}^{\infty} \frac{e^{-\kappa u^{2} t} C_{0}(u r, u a) d u}{u\left(u^{2}+n^{2} \pi^{2} / l^{2}\right)\left[J_{0}^{2}(u a)+Y_{0}^{2}(u a)\right]}\right\}
\end{aligned}
$$

where

$$
C_{0}(x, y)=J_{0}(x) Y_{0}(y)-Y_{0}(x) J_{0}(y) .
$$

5. The region $r>a, z>0 ; z=0$ kept at unity for $t>0, r>a ; r=a$ kept at zero for $z>0, t>0$; the initial temperature zero. Here

$$
\bar{v}=\frac{1}{p} e^{-q z}-\frac{2}{\pi p} \int_{0}^{\infty} \frac{u \sin u z}{u^{2}+q^{2}} \frac{K_{0}\left(r q^{\prime}\right)}{K_{0}\left(a q^{\prime}\right)} d u,
$$

where $q$ and $q^{\prime}$ are defined in (7) and (18) respectively, satisfies the subsidiary equation and its boundary condition at $z=0$. It also satisfies the boundary condition at $r=a$ since

$$
\frac{2}{\pi} \int_{0}^{\infty} \frac{u \sin u z}{u^{2}+q^{2}} d u=e^{-q z} .
$$

Using the inversion theorem we obtain from (30)

$$
\begin{array}{r}
v=1-\frac{2}{\pi} \int_{0}^{\infty} \frac{K_{0}(u r) \sin u z}{u K_{0}(u a)} d u+\frac{4}{\pi^{2}} \int_{0}^{\infty} e^{-\kappa u^{2} t} u \sin u z d u \\
\cdot \int_{0}^{\infty} \frac{e^{-\kappa u^{\prime 2} t} C_{0}\left(r u^{\prime}, a u^{\prime}\right) d u^{\prime}}{u^{\prime}\left(u^{\prime 2}+u^{2}\right)\left[J_{0}^{2}\left(a u^{\prime}\right)+Y_{0}^{2}\left(a u^{\prime}\right)\right]} .
\end{array}
$$

6. The region $r>a, z>0 ; r=a$ kept at unity for $z>0, t>0 ; z=0$ kept at zero for $r>a, t>0$; the initial temperature zero. Here 


$$
\bar{v}=\frac{1}{p} \frac{K_{0}(q r)}{K_{0}(q a)}+\frac{2}{\pi p} \int_{0}^{\infty} e^{-z q^{\prime}} \frac{C_{0}(u r, u a) u d u}{\left(q^{2}+u^{2}\right)\left[J_{0}^{2}(u a)+Y_{0}^{2}(u a)\right]}
$$

satisfies the subsidiary equation and its boundary condition at $r=a$. It also satisfies the boundary condition at $z=0$, since, by Weber's integral theorem,

$$
\frac{K_{0}(q r)}{K_{0}(q a)}+\frac{2}{\pi} \int_{0}^{\infty} \frac{C_{0}(u r, u a) u d u}{\left(q^{2}+u^{2}\right)\left[J_{0}^{2}(u a)+Y_{0}^{2}(u a)\right]}=0 .
$$

It follows that

$$
\begin{aligned}
v=1 & +\frac{2}{\pi} \int_{0}^{\infty} \frac{e^{-u z} C_{0}(r u, a u) d u}{u\left[J_{0}^{2}(a u)+Y_{0}^{2}(a u)\right]} \\
& +\frac{4}{\pi^{2}} \int_{0}^{\infty} \frac{e^{-\kappa u^{2} t} C_{0}(r u, a u) u d u}{J_{0}^{2}(a u)+Y_{0}^{2}(a u)} \int_{0}^{\infty} \frac{e^{-\kappa u^{\prime 2} t} \sin u^{\prime} z d u^{\prime}}{u^{\prime}\left(u^{2}+u^{\prime 2}\right)} .
\end{aligned}
$$

7. The finite cylinder and similar problems. In this section four results are given for completeness which are obtained by the method of $\$ 2$; these problems may also be solved by the method of $\$ 3$, and in all cases the transient parts of the solutions are identical, but the steady state parts are obtained in a different form.

(i) The region $0 \leqq r<a, z>0 ; z=0$ kept at unity for $t>0,0 \leqq r<a$; $r=a$ kept at zero for $z>0, t>0$; the initial temperature zero:

$$
\bar{v}=\frac{1}{p} e^{-q z}-\frac{2}{\pi p} \int_{0}^{\infty} \frac{u \sin u z}{q^{2}+u^{2}} \frac{I_{0}\left(r q^{\prime}\right)}{I_{0}\left(a q^{\prime}\right)} d u,
$$

where $q$ and $q^{\prime}$ are defined in (7) and (18);

$$
\begin{aligned}
v=1 & -\frac{2}{\pi} \int_{0}^{\infty} \frac{I_{0}(u r) \sin u z d u}{u I_{0}(u a)} \\
& -\frac{4}{\pi a} \sum_{m=1}^{\infty} \frac{e^{-\kappa \alpha_{m}^{2} t} J_{0}\left(r \alpha_{m}\right)}{\alpha_{m} J_{1}\left(a \alpha_{m}\right)} \int_{0}^{\infty} \frac{e^{-\kappa u^{2} t} u \sin u z d u}{\left(\alpha_{m}^{2}+u^{2}\right)},
\end{aligned}
$$

where the $\alpha_{m}$ are the positive roots of $J_{0}(a \alpha)=0$.

(ii) The region $r>a, 0<z<l ; r=a$ kept at unity for $t>0,0<z<l$; the other surfaces kept at zero for $t>0$; the initial temperature zero:

$$
\bar{v}=\frac{K_{0}(q r)}{p K_{0}(q a)}+\int_{0}^{\infty} \frac{2 \cosh q^{\prime}\left(\frac{1}{2} l-z\right) C_{0}(r u, a u) u d u}{\pi p q^{\prime 2} \cosh \frac{1}{2} q^{\prime} l\left[J_{0}^{2}(u a)+Y_{0}^{2}(u a)\right]},
$$




$$
\begin{aligned}
& v=1+ \frac{2}{\pi} \int_{0}^{\infty} \frac{\cosh u\left(\frac{1}{2} l-z\right) C_{0}(r u, a u) d u}{u \cosh \frac{1}{2} l u\left[J_{0}^{2}(a u)+Y_{0}^{2}(a u)\right]} \\
&+\frac{8}{\pi^{2}} \sum_{n=0}^{\infty}\left\{e^{-\kappa(2 n+1)^{2} \pi^{2} t / l^{2}} \frac{\sin (2 n+1) \pi z / l}{(2 n+1)}\right. \\
&\left.\qquad \int_{0}^{\infty} \frac{e^{-\kappa u^{2} t} C_{0}(r u, a u) u d u}{\left[u^{2}+(2 n+1)^{2} \pi^{2} / l^{2}\right]\left[J_{0}^{2}(a u)+Y_{0}^{2}(a u)\right]}\right\} .
\end{aligned}
$$

(iii) The finite cylinder $0 \leqq r<a, 0<z<l ; r=a$ kept at unity and the other surfaces at zero for $t>0$; the initial temperature zero:

$$
\bar{v}=\frac{I_{0}(q r)}{p I_{0}(q a)}-\frac{2}{a} \sum_{m=1}^{\infty} \frac{\alpha_{m} J_{0}\left(r \alpha_{m}\right)}{p q_{m}^{2} J_{1}\left(a \alpha_{m}\right)} \frac{\cosh \left(\frac{1}{2} l-z\right) q_{m}}{\cosh \frac{1}{2} l q_{m}}
$$

where $\alpha_{m}, m=1,2, \cdots$, are the positive roots of $J_{0}(a \alpha)=0$ and $q_{m}=\left(q^{2}+\alpha_{m}^{2}\right)^{1 / 2}$;

$$
\begin{aligned}
& v=1-\frac{2}{a} \sum_{m=1}^{\infty} \frac{J_{0}\left(r \alpha_{m}\right)}{\alpha_{m} J_{1}\left(a \alpha_{m}\right)} \frac{\cosh \left(\frac{1}{2} l-z\right) \alpha_{m}}{\cosh \frac{1}{2} l \alpha_{m}} \\
&- \frac{8}{a \pi} \sum_{m=1}^{\infty}\left\{\frac{\alpha_{m} J_{0}\left(r \alpha_{m}\right)}{J_{1}\left(a \alpha_{m}\right)} e^{-\kappa \alpha_{m}^{2} t}\right. \\
&\left.\cdot \sum_{n=0}^{\infty} \frac{e^{-\kappa(2 n+1)^{2} \pi^{2} t / l^{2}} \sin (2 n+1) \pi z / l}{(2 n+1)\left[\alpha_{m}^{2}+(2 n+1)^{2} \pi^{2} / l^{2}\right]}\right\} .
\end{aligned}
$$

(iv) The finite cylinder $0 \leqq r<a, 0<z<l ; z=l$ kept at unity and the other surfaces at zero for $t>0$; the initial temperature zero:

$$
\bar{v}=\frac{\sinh q z}{p \sinh q l}+\frac{2 \pi}{p l^{2}} \sum_{n=1}^{\infty} \frac{n(-)^{n} I_{0}\left(r q_{n}\right)}{q_{n}^{2} I_{0}\left(a q_{n}\right)} \sin \frac{n \pi z}{l},
$$

where $q_{n}=\left(q^{2}+n^{2} \pi^{2} / l^{2}\right)^{1 / 2}$;

$$
\begin{aligned}
v=\frac{z}{l} & +\frac{2}{\pi} \sum_{n=1}^{\infty} \frac{(-)^{n} I_{0}(n \pi r / l)}{n I_{0}(n \pi a / l)} \sin \frac{n \pi z}{l} \\
& +\frac{4 \pi}{a} \sum_{n=1}^{\infty} n(-)^{n} \sin \frac{n \pi z}{l} \sum_{m=1}^{\infty} \frac{J_{0}\left(r \alpha_{m}\right) e^{-\kappa t\left(\alpha_{m}^{2}+n^{2} \pi^{2} / l^{2}\right)}}{\alpha_{m}\left(l^{2} \alpha_{m}^{2}+n^{2} \pi^{2}\right) J_{1}\left(a \alpha_{m}\right)},
\end{aligned}
$$

where $\alpha_{m}, m=1,2, \cdots$, are the positive roots of $J_{0}(a \alpha)=0$.

The University of Tasmania,

Hobart, TASMania 